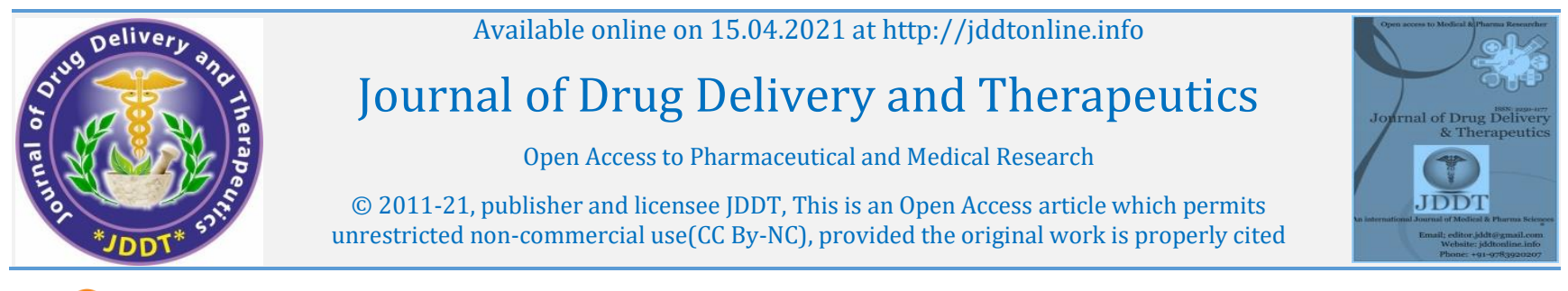

Open Access Full Text Article

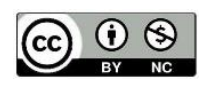

Case Report

\title{
Clinical diagnostic and treatment of COVID 19 of bilateral pneumonia:
}

\section{Case report}

\author{
Otljanski A, Kipevska K \\ University Hospital of Surgery "SvNaumOhridski", Ss. Cyril and Methodius University, Faculty of Medicine, Skopje North Macedonia
}

\section{Article Info:

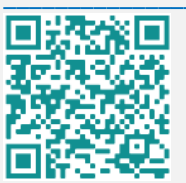 \\ Article History: \\ Received 21 Feb 2021; \\ Review Completed 14 March 2021 Accepted 19 March 2021; \\ Available online 15 April 2021}

Cite this article as:

Otljanski A, Kipevska K, Clinical diagnostic and treatment of COVID 19 of bilateral pneumonia: Case report, Journal of Drug Delivery and Therapeutics. 2021; $11(2-$ s):1-3

DOI: http://dx.doi.org/10.22270/jddt.v11i2-s.4629

\section{Abstract}

In 2019 year the world was attacked with corona virus (SARS-CoV-2) and in march 2020, the World Health Organization (WHO) declared the COVID-19 outbreak a pandemic.The symptoms and clinical state in this COVID-19 infection was different, from asymptomatic to mild and severe symptoms, which presented different form of disease: mild, moderate or severe, with or without complications. We presented case with COVID 19 pneumonia as one of the form of COVID infection, with diagnosis, treatment and all investigations we done in our hospital. With complex treatment of this virus infection we successful improved the clinical state of patients with COVID 19 pneumonia.

Keywords: coronavirus, SARS-CoV-2, COVID-19, COVID 19 pneumonia

\section{*Address for Correspondence:}

Otljanski Aleksandar, Specialist, Internal Medicine in University Hospital of Surgery "SvNaumOhridski", Ss. Cyril and Methodius University, Faculty of Medicine, Skopje North Macedonia

\section{INTRODUCTION}

COVID 19 is a new type of viral infection emerged in Wuhan City, China, and genomic sequencing data of this virus, suggesting a novel CoV strain (2019-nCoV), which provokes different form of clinical manifestation, but the most often was severe acute respiratory syndrome CoV-2 (SARS-CoV2). COVID-19 shows less severe pathogenescompared to diseases caused by previously known human CoVs but with higher transmission competence. Asa result of this the number of confirmed cases continuously increasing globally. ${ }^{1,2,3}$

COVID-19 affects people in different ways. Most infected people will develop the heterogeneous of symptoms with mild to moderate illness and recover with or without hospitalization. The most common symptoms are fever, dry cough, tiredness, but there are other symptoms that develop in COVID-19 like conjunctivitis, a rash on skin, or discolouration of fingers or toes. Despite of clinical state some symptoms may be very serious like difficulty breathing or shortness of breath, chest pain, loss of speech or movement.4,5 The severity of COVID-19 symptoms can range from very mild to severe. There are no rules which people have only a few symptoms, some people may have no symptoms and others developed as worsened shortness of breath and pneumonia, about a week after symptoms start. People with comorbidites seems to be more like to develop mild or severe form of COVID-19.
COVID 19 pneumonia was first noticed at the end of 2019 in the patients in Wuhan, China. It causes atypical pneumonia that manifest itself with dry cough, fever and shortness of breath. According to research in COVID 19 CT lung scan is more likely to find pneumonia on both lungs instead of one and lungs that had characteristic "ground glass" appearance. People who are at most risk for COVID 19 pneumonia are people older than 65 years old, people with chronic lung diseases and people with weakened immune system. There is no specific treatment for COVID 19 pneumonia. Most commonly used treatments are focused on supportive care, often receiving oxygen therapy and antibiotics for prevention of secondary bacterial infection. ${ }^{6}$

\section{CASE REPORT}

A 55 years old patient was admitted to the hospital with the symptoms of cough and tiredness that started 7 days ago and difficulty breathing that started one day before coming to hospital. On admission the patient was subfebrile $37.5 \mathrm{C}$, blood pressure was $120 / 80 \mathrm{mmHg}$, heart rate was $105 / \mathrm{min}$, ECG was normal and oxygen saturation was $84 \%$. The patient did not have any comorbidities and his BMI was normal. Laboratory analyses on admission showed values of $\mathrm{Hb} 142 \mathrm{~g} / \mathrm{l}$, Le 4.8, Lymph 1.7, Tr 182, serum iron 2.6 umol/l, CRP $136 \mathrm{mg} /$ L.CT scan on lungs showed subpleural ground glass opacity bilateral predominantly in posterior segments. 


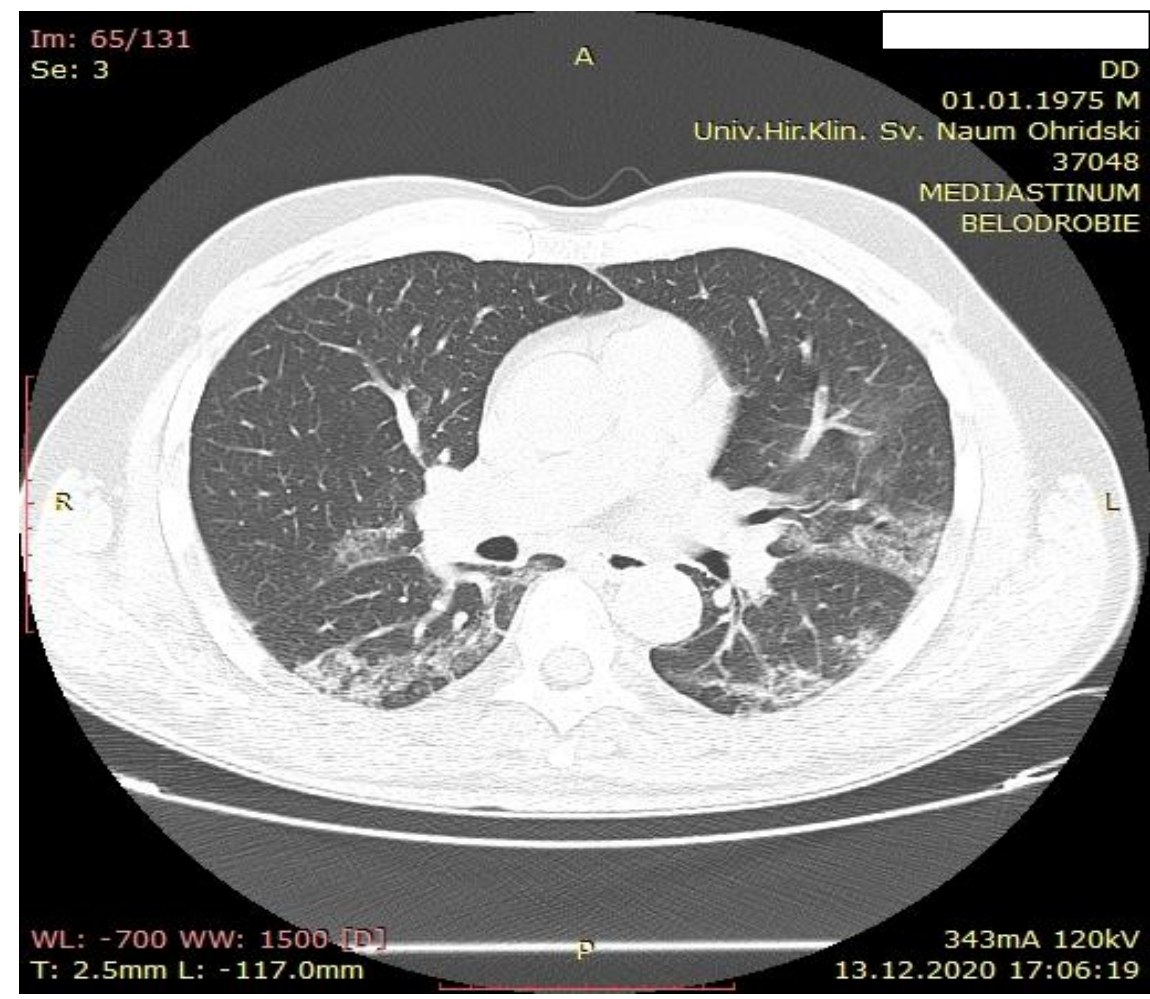

Figure 1: Thoracic computed tomography showing bilateral ground-glass opacities

Hemostatic parameters of the patient at admission were Ddimers $912 \mathrm{ngr} / \mathrm{ml}$, normal prothrombin time and normal partial thromboplastin time. Inflammatory markers were with the values of ferritin $760.89 \mathrm{ug} / \mathrm{L}$, interleukin 6 $9.91 \mathrm{pg} / \mathrm{ml}$, procalcitonin $0.056 \mathrm{ng} / \mathrm{ml}$. PCR test was negative, but COVID antibodies showed high values of COVID 19 IgM $5.87 \mathrm{AU} / \mathrm{ml}$ and COVID 19 IgG $6.59 \mathrm{AU} / \mathrm{ml}$.

Treatment -During the hospitalization of the patient we consulted infectious disease specialist and transfusiology specialist and they suggested treatment with meropenem, enoxaparin, favipiravir, intravenous corticosteroid and oxygen treatment with $5 \mathrm{~L} / \mathrm{min}$. The dose they suggest are: Favipiravir in dosage of $1600 \mathrm{mg}$ first day and $600 \mathrm{mg}$ from day two to day five. Urbason was given in dosage $80+40 \mathrm{mg} 3$ days, then $40+40 \mathrm{mg} 3$ days, then only $40 \mathrm{mg}$ daily for four days.

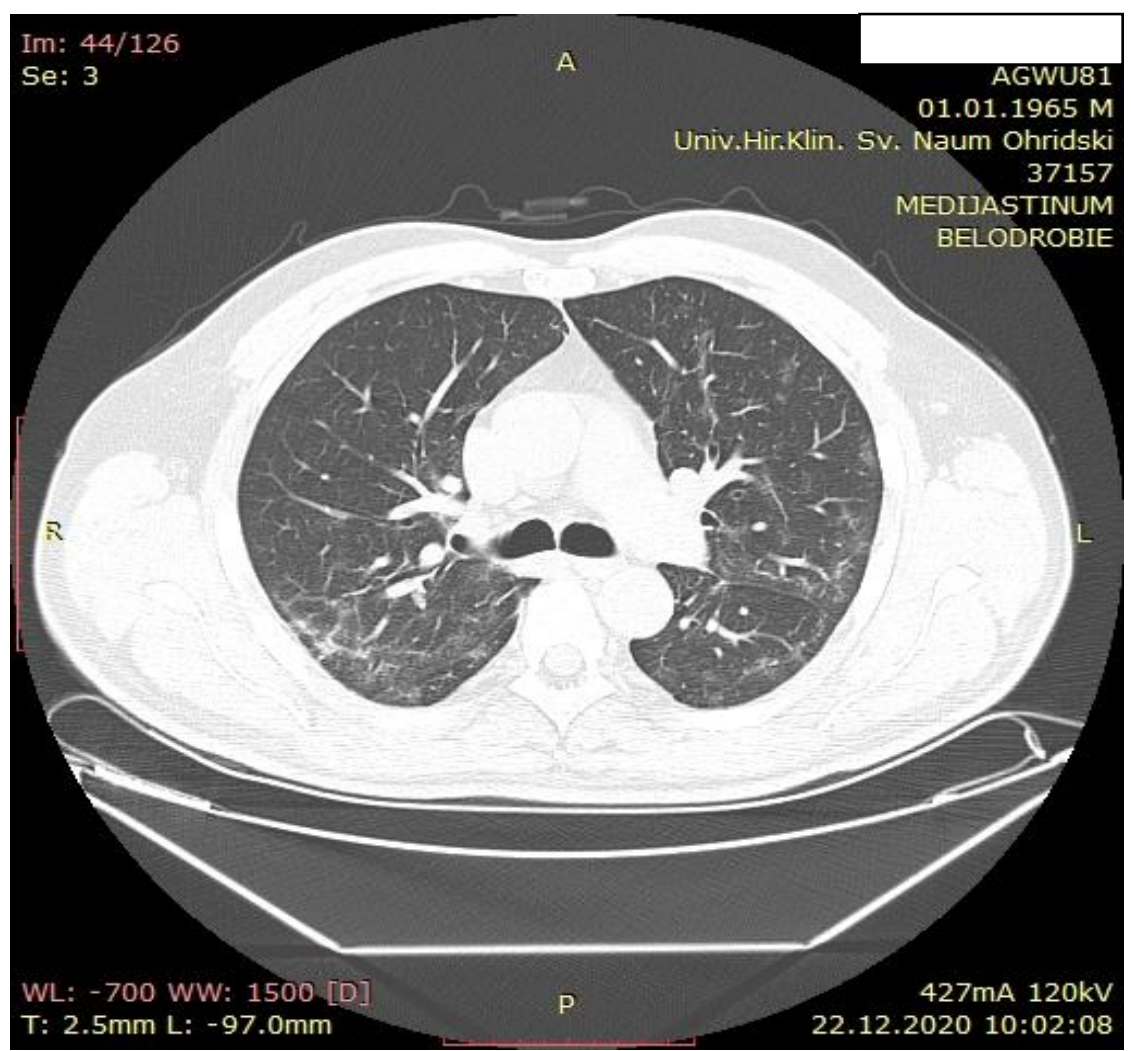

Figure 2: Thoracic computed tomography after 9 days of treatement 
On the third day of the treatment another antibiotic was given per os, moxifloxacin once a day. After fifth day of treatment patient showed significant improvement with saturation $92 \%$ on nasal mask oxygen $21 /$ min and without oxygen mask saturation $90 \%$. We take laboratory analysis on fifth day and they showed Le 9.8, lymph 1.3, Tr 377, Fe 15.3.CRP 12.3, ferritin 745, d-dimers 933. After eight days of treatment saturation was $96 \%$ without nasal mask. Laboratory analysis on ten day of hospitalization was Le 8.9, $\operatorname{Tr} 372$, lymph 2.2, Fe 20.4, CRP 1.9, D-dimers 862. We want to see the effect of treatment and we done new CT scan on the lungs which show the significant improvement on ground glass opacity and small fibrous changes.

The treatment with antibiotics and corticosteroids was finished after ten days.

After 15 days of treatment patient was discharge with recommendation of transfusiologist for Amp. Enoxaparin 0.4 once a day for one more week. After 7 days of release he came in ambulance feeling much better, starting to do working at home and walking outside on small distance.His control D-dimer were $1006 \mathrm{ngr} / \mathrm{mL}$ and after consultation with transfusiologist anticoagulant treatment was stopped and he was given antiplatelet drug with Tbl. Acetylsalicylic acid $100 \mathrm{mg} 1 \mathrm{x} 1$.

\section{DISCUSSION}

In this article we present case of COVID-19 pneumonia, as a one of the manifestation of COVID-19 infection. Coronaviruses are a family of viruses that can cause severe acute respiratory syndrome (SARS) and Middle East respiratory syndrome (MERS) but in 2019, the world was attackedwith a new coronavirus that originated in China. 6,7

The virus is known as the severe acute respiratory syndrome coronavirus 2 (SARS-CoV-2) and the disease it causes is called coronavirus disease 2019 (COVID-19) and in March 2020, the World Health Organization (WHO) declared the COVID-19 outbreak a pandemic. This situation was new for every doctor in the world from aspect of diagnosis, treatment and complication from this pandemic. The first was note that people who are older have a higher risk of serious illness from COVID-19, people with existing chronic medical conditions like serious heart diseases, such as heart failure, coronary artery disease or cardiomyopathy, chronic obstructive pulmonary disease (COPD, Type 2 diabetes, obesity or severe obesity, cancer, weakened immune system etc). In our case with COVID 19 pneumonia we doneefficacy diagnosis, laboratory tests, CT scan on the lungs and complex treatment with follow up of patient after discharge and improvement the clinic state of patient.8,9,10

\section{CONCLUSION}

COVID 19 pneumonia was one of the form of COVID 19 infection. This form of treatments are focused on supportive care, often receiving oxygen therapy and antibiotics for prevention of secondary bacterial infection.In our case thetreatment of hospitalized patient with COVID 19 bilateral pneumonia with antiviral medication, antibiotics, corticosteroids, oxygen and anticoagulation therapy, showed efficiency of treatment and stabilization of clinic state of patient.

\section{REFERENCES}

1. Wang C, Horby PW, Hayden FG, Gao GF. A novel coronavirus outbreak of global health concern.Lancet. 2020 Feb 15; 395(10223):470-473.

2. Cate Wood. Infections without borders: a new coronavirus in Wuhan, China.Br J Nurs.. 2020 Feb 13; 29(3):166-167.

3. Dhama K, Khan S, Tiwari R, Sircar S, Bhat S, Malik YS, Singh KP, Chaicumpa W, D Bonilla-Aldana K, Rodriguez-Morales AJ Coronavirus Disease 2019-COVID-19.ClinMicrobiol Rev. 2020 Jun 24; 33(4):e00028-20

4. Huang C, Wang Y, Li X, Ren L, Zhao J, Hu Y, Zhang L, Fan G. Clinical features of patients infected with 2019 novel coronavirus in Wuhan, China.Lancet. 2020 Feb 15; 395(10223):497-506.

5. Cascella M, Rajnik M, Cuomo A, Dulebohn SC, Raffaela Di Napol Features, Evaluation, and Treatment of Coronavirus.StatPearls, October 4, 2020.

6. Seibert FS, Toma D, Bauer F, Paniskaki K, Detection of SARS-CoV-2 pneumonia: two case reports. Journal of Medical Case Reports. 2020, volume 14, Article number: 242.

7. Corman VM, Landt O, Kaiser M, Molenkamp R, Meijer A, Chu DKW, Bleicker T, Brunink S, Schneider J, Schmidt ML, et al. Detection of 2019 novel coronavirus (2019-nCoV) by real-time RT-PCR Euro Surveill. 2020; 25:2.

8. Sanders JM, Monogue ML, Jodlowski TZ, Cutrell JB. Pharmacologic Treatments for Coronavirus Disease 2019 (COVID-19): A Review. JAMA. 2020 Apr 13

9. Zarogoulidis P, Papanas N, Kioumis I, Chatzaki E, Maltezos E, Zarogoulidis K. Macrolides: from in vitro anti-inflammatory and immunomodulatory properties to clinical practice in respiratory diseases. Eur J Clin Pharmacol. 2012 May; 68(5):479-503

10. Kollias A, Kyriakoulis KG, Dimakakos E, Poulakou G, Stergiou GS, Syrigos K. Thromboembolic risk and anticoagulant therapy in COVID-19 patients: emerging evidence and call for action. Br J Haematol. 2020 Jun; 189(5):846-847. 